Nota: a versão corrigida dos artigos abaixo encontra-se disponível, em texto integral, na biblioteca eletrônica SciELO (www.scielo.br).

Errata de

\title{
Globalização, dilemas dos comuns e qualidade de vida sustentável: do que precisamos, o que podemos fazer, o que podemos conseguir?
}

[Estudos de Psicologia, 8(2), 221-234]

Charles Vlek

Universidade de Groningen, Holanda

E-mail: c.a.j.vlek@ppsw.rug.nl

Estudos de Psicologia lamenta que no artigo acima a Tabela 5 tenha sido publicada incorretamente.

A forma correta é agora reproduzida abaixo.

Tabela 5

Exigências dos ministros da Organização para Cooperação e Desenvolvimento Econômico para um transporte sustentável.

- Satisfação das necessidades básicas de acesso de indivíduos, companhias e sociedades...

- Seguro, saudável e ambientalmente amigável ...

- Eqüitativo intra e inter gerações ...

- Acessível financeiramente, justo e eficientemente operado ...

- Protagonista de uma economia competitiva ...

- Permitindo desenvolvimento regional equilibrado ...

- Utilizando, de maneira otimizada, energia e materiais renováveis ...

- Uso controlado de não-renováveis ...

- Minimizando uso da terra ...

- Minimizando geração de ruídos ... 
Errata de

\section{A Teoria das Facetas: noções básicas}

[Estudos de Psicologia, 8(3), 357-365]

\section{Wolfgang Bilsky}

Westfälische Wilhelms-Universität Münster

E-mail: bilsky@psy.uni-muenster.de

Estudos de Psicologia lamenta que no artigo acima a Figura 5 tenha sido publicada incorretamente.

A forma correta é agora reproduzida abaixo.

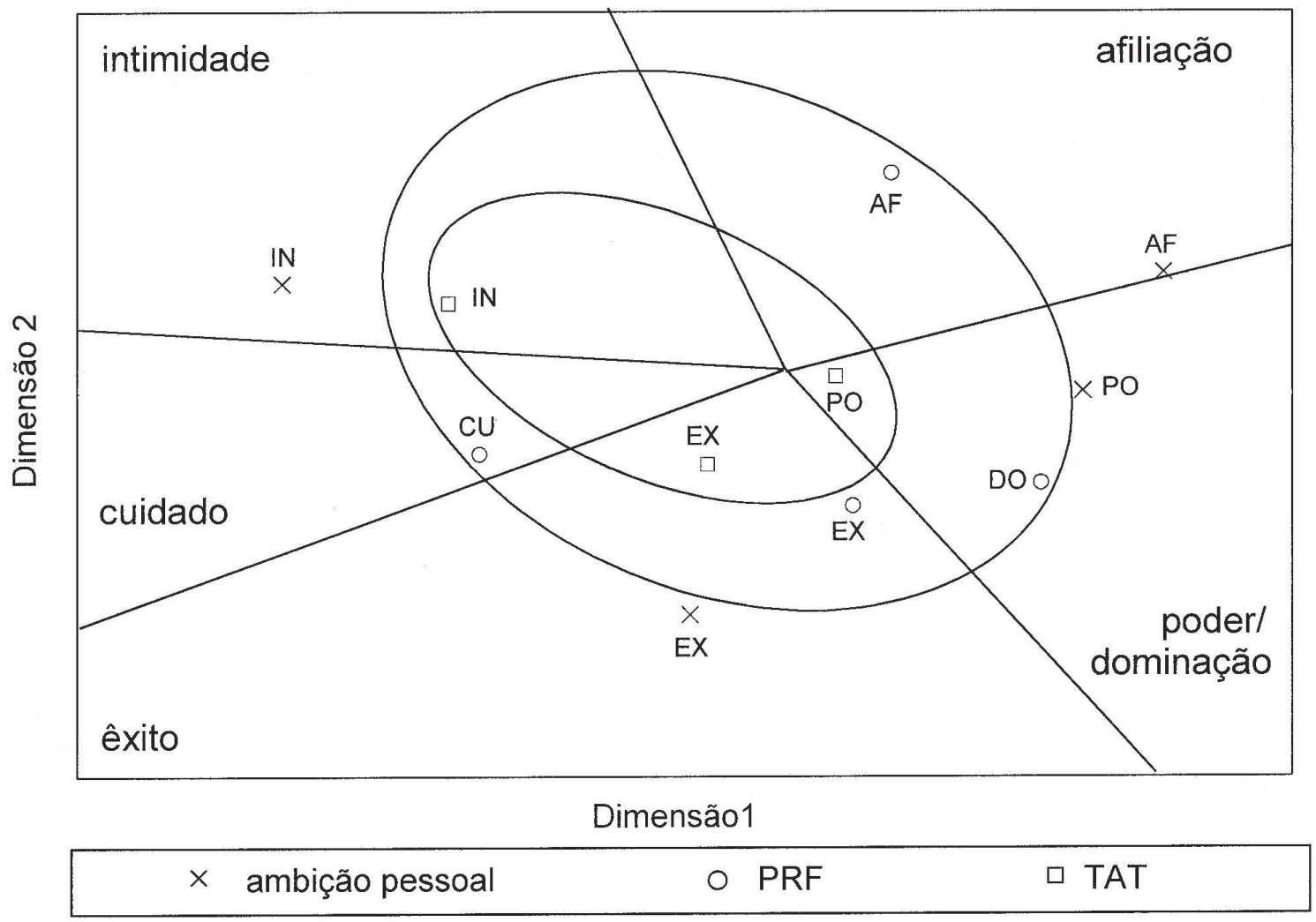

Figura 5. Radex: relação entre diferentes tipos de motivos (Emmons \& McAdams, 1991; ver Bilsky, 1999a). 
Errata de

\title{
Socioeconomic status in Brazilian psychological research: I. validity, measurement, and application
}

[Estudos de Psicologia, 8(3), 375-383]

\author{
Rodolfo de Castro Ribas Jr. \\ Universidade Federal do Rio de Janeiro \\ E-mail: rribas@ufrj.br \\ Maria Lucia Seidl de Moura \\ Universidade do Estado do Rio de Janeiro \\ Isabela Dias Soares \\ Alessandra Aparecida do Nascimento Gomes \\ Universidade Federal do Rio de Janeiro \\ Marc H. Bornstein \\ National Institute of Child Health and Human Development - USA
}

Estudos de Psicologia lamenta que no artigo acima a Tabela 2 tenha sido publicada incorretamente.

A forma correta é agora reproduzida abaixo.

Table 2

Percentage of articles published by journal from 1998 to 2000 that employed the term "socioeconomic status" and unstandardized Beta Coefficients for regression analyses predicting percentage of articles that employed the term socioeconomic status as a function of year for selected journals

\begin{tabular}{lrrr}
\hline \multicolumn{1}{c}{ Journal } & $\%$ & B & SE B \\
\hline Journal of Family Psychology & 22.94 & 3.19 & $.55^{*}$ \\
Developmental Psychology & 22.91 & .90 & $.30 *$ \\
Health Psychology & 19.89 & .74 & .92 \\
Journal of Consulting and Clinical Psychology & 19.00 & .18 & .15 \\
Journal of Abnormal Psychology & 17.16 & .81 & .40 \\
Journal of Educational Psychology & 16.77 & .98 & $.37 *$ \\
Psychological Assessment & 14.08 & .25 & .40 \\
Psychological Bulletin & 13.76 & .23 & .34 \\
Psychology and Aging & 12.64 & .13 & .52 \\
Journal of Counseling Psychology & 12.18 & .69 & $.28 *$ \\
Journal of Personality and Social Psychology & 6.83 & .22 & .15 \\
American Psychologist & 6.23 & .13 & .13 \\
\hline
\end{tabular}

$* p<.05$. 
Errata de

\title{
Socioeconomic status in Brazilian psychological research: I. validity, measurement, and application
}

[Estudos de Psicologia, 8(3), 375-383]

\author{
Rodolfo de Castro Ribas Jr. \\ Universidade Federal do Rio de Janeiro \\ E-mail: rribas@ufrj.br \\ Maria Lucia Seidl de Moura \\ Universidade do Estado do Rio de Janeiro \\ Isabela Dias Soares \\ Alessandra Aparecida do Nascimento Gomes \\ Universidade Federal do Rio de Janeiro \\ Marc H. Bornstein \\ National Institute of Child Health and Human Development - USA
}

Estudos de Psicologia lamenta que no artigo acima a Tabela 2 tenha sido publicada incorretamente.

A forma correta é agora reproduzida abaixo.

Table 2

Zero-order correlations among the variables

\begin{tabular}{|c|c|c|c|c|c|c|c|c|c|}
\hline Variable & 1 & 2 & 3 & 4 & 5 & 6 & 7 & 8 & 9 \\
\hline 1. Father's Education ${ }^{\mathrm{a}}$ & - & & & & & . & & & \\
\hline 2. Father's Occupation & $.74^{* *}$ & - & & & & & & & \\
\hline 3. Father's Age (years) & $.42 * *$ & $.40 * *$ & - & & & & & & \\
\hline 4. Mother's Education ${ }^{\mathrm{a}}$ & $.69^{* *}$ & $.71 * *$ & $.44^{* *}$ & - & & & & & \\
\hline 5. Mother's Occupation ${ }^{\mathrm{b}}$ & $.62 * *$ & $.71 * *$ & .29 & $.74 * *$ & - & & & & \\
\hline 6. Mother's Age (years) & $.41 * *$ & $.47 * *$ & $.63 * *$ & $.52 * *$ & $.40 *$ & - & & & \\
\hline 7. $\mathrm{HI}^{\mathrm{c}}$ & $.82 * *$ & $.94 * *$ & $.44 * *$ & $.82 * *$ & $.90 * *$ & $.52 * *$ & - & & \\
\hline 8. SIOP $^{d}$ & $.67^{* *}$ & $.87 * *$ & $.34 * *$ & $.75 * *$ & $.85^{* *}$ & $.54 * *$ & $.89^{* *}$ & - & \\
\hline 9. ISEI $^{\mathrm{e}}$ & $.75^{* *}$ & $.89 * *$ & $.41 * *$ & $.78 * *$ & $.85^{* *}$ & $.49 * *$ & $.94 * *$ & $.90 * *$ & - \\
\hline 10. $\mathrm{KIDI}^{\mathrm{f}}$ & $.35^{*}$ & $.34 * *$ & .07 & $.41 * *$ & .24 & .01 & $.36^{* *}$ & $.28^{*}$ & $.31 *$ \\
\hline
\end{tabular}

${ }^{a}$ Hollingshead 7-point education scale. ${ }^{b}$ Hollingshead 9-point occupation scale.

${ }^{c}$ Hollingshead Four-Factor Index of Social Status scale. ${ }^{\mathrm{d}}$ Standard International Occupational Prestige Scale. ${ }^{\mathrm{e}}$ International Socio-Economic Index of Occupational Status. ${ }^{\mathrm{f}}$ Knowledge of the Infant Development Inventory.

$* p<.05$. ** $p<.01$. 